Portland State University

PDXScholar

Electrical and Computer Engineering Faculty

Publications and Presentations

7-1-1992

\title{
New speckle technique for noncontact measurement of small creep rates
}

\author{
Donald D. Duncan \\ Portland State University \\ F. Fausten Mark \\ Lawrence W. Hunter
}

Follow this and additional works at: https://pdxscholar.library.pdx.edu/ece_fac

Part of the Electrical and Computer Engineering Commons

Let us know how access to this document benefits you.

\section{Citation Details}

D. D. Duncan, F. F. Mark, L. W. Hunter, "A New Speckle Technique for Noncontact Measurement of Small Creep Rates," Optical Engineering, V. 31, pp. 1583-1589 (1992).

This Article is brought to you for free and open access. It has been accepted for inclusion in Electrical and Computer Engineering Faculty Publications and Presentations by an authorized administrator of PDXScholar. Please contact us if we can make this document more accessible: pdxscholar@pdx.edu. 


\section{New speckle technique for noncontact measurement of small creep rates}

\author{
Donald D. Duncan \\ F. Fausten Mark \\ Lawrence W. Hunter \\ Johns Hopkins University Applied Physics \\ Laboratory \\ Johns Hopkins Road \\ Laurel, Maryland 20723-6099
}

\begin{abstract}
A highly sensitive method for measuring thermal expansion, mechanical strain, and creep rates has been developed. This technique is based on Yamaguchi's speckle strain gauge concept, but uses a novel data-processing approach that provides estimates of the time rate of inplane strain. The approach is appropriate for assessing very small strain rates in hostile environments. It provides simultaneous global estimates of the strain at both small and large gauge sizes. This may be of importance in studying materials with different short- and long-range orders. General advantages of the technique are compact design, modest resolution requirements, insensitivity to surface microstructure changes (as seen with oxidation), and relative insensitivity to zero-mean noise processes such as turbulence and vibration.
\end{abstract}

Subject terms: strain rates; speckle metrology.

Optical Engineering 31(7), 1583-1589 (July 1992).

\section{Background}

Creep is the slow deformation that occurs in materials under static loads and is important at high temperatures. It can result in the delamination of protective ceramic coatings and the growth of cracks. Typical creep rates (fractional deformation per unit time) for ceramics are $0.2 \mathrm{~h}^{-1}$ for silicon dioxide glass and $10^{-6} \mathrm{~h}^{-1}$ for polycrystalline alumina, both at $1300^{\circ} \mathrm{C}$ ad $1800 \mathrm{psi}$; the rate of creep increases as stress and temperature rise. Mechanisms of creep include grain-boundary deformation, dislocation movements, and diffusion. Therefore, there is no simple predictive model of creep, and measurement methods take on added importance. Measurements can be challenging at ultrahigh temperatures where remote techniques are needed to avoid interference from deformation in the clamps that apply the stress to the specimen. The long time required to observe creep in some ceramics adds to the challenge, as does the variability of many advanced materials, which necessitates many repetitive measurements on many specimens. Our laser speckle strain gauge technique allows an accurate noncontact measurement of the creep characteristics of these materials at high temperatures.

\section{Experimental Configuration}

The concept behind our experimental configuration, which is the same as that described by Yamaguchi, ${ }^{1}$ is illustrated in Fig. 1. It uses the phenomenon of "objective" or nonimaged laser speckle ${ }^{2}$ to determine thermal expansion, mechanical strain, and creep rates. The " $\mathrm{dog}$ bone" represents the specimen under tensile stress. It is illuminated normally

Paper 17111 received Nov. 22, 1991; revised manuscript received Jan. 24, 1992; accepted for publication Jan. 25, 1992 .

(C) 1992 Society of Photo-Optical Instrumentation Engineers. 0091-3286/92/\$2.00. with a laser beam and the illuminated portion of the specimen constitutes the gauge size. Symmetrically opposed at angle $\theta$ with respect to the specimen normal, and in the plane that contains the incident laser beam, are a pair of linear array detectors. The difference in the speckle translation (before and after stress), as measured by these two detectors is given by the formula

$\delta A_{x}=-2 L \varepsilon_{x x} \tan \theta-2 a_{z} \sin \theta$,

where

$L=$ distance between specimen and detectors

$\varepsilon_{x x}=$ linear strain in the plane of the specimen

$a_{z}=$ out-of-plane movement.

A proper choice of observation distance and angle can make the differential speckle pattern movement $\delta A_{x}$ insensitive to the out-of-plane movement. Effects due to all other rigid body motions are eliminated by the differential technique. This general measurement scheme has the advantage that it is simple to set up and easy to calibrate. This latter point is an important advantage over alternative techniques. ${ }^{3,4}$

With this procedure, using a very basic measure of speckle position, Yamaguchi ${ }^{5,6}$ has demonstrated a sensitivity of 10 microstrain $(10 \mu \varepsilon)$. A variation on Yamaguchi's technique is also in use by Barranger ${ }^{7}$ for measurement of creep of furnace-heated specimens. Takemori et al.$^{8}$ have recently demonstrated strain resolutions on the order of 1 microstrain, which they obtained by interpolating the correlation function between the reference exposure and the sample exposure. Another technique is to interpolate the speckle pattern itself. ${ }^{9}$ Improved resolutions obtained by these means, however, exact a price: small gauge sizes. Moreover, adequately assessing the spatial statistics of creep requires large gauge sizes. 


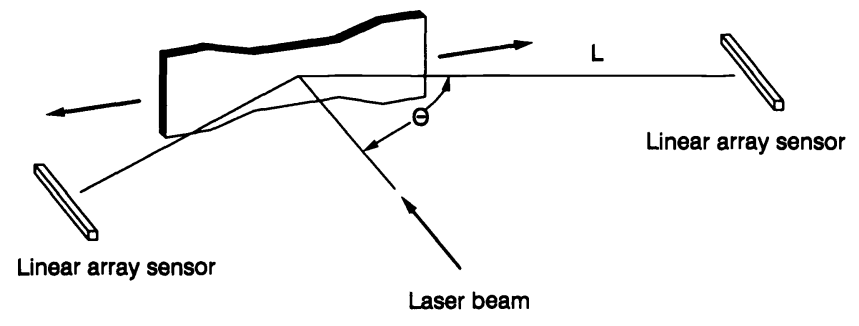

Fig. 1 Configuration for instruments used in measurement technique.

For objective speckle, Goodman ${ }^{10}$ has shown that the minimum scale size is on the order of

$\sigma \sim \frac{\lambda L}{D}$

where $\lambda$ is the wavelength, $L$ is the observation distance, and $D$ is the size of the (uniformly) illuminated spot on the subject. Conversely, the large-scale structure in the scattered light is determined by the fine-scale structure of the object surface. ${ }^{11}$ For reasons that will become apparent later, one should sample this speckle pattern at or above the Nyquist rate. That is, one chooses $p \leqslant \sigma / 2$, where $p$ is the detector pixel pitch (the center-to-center distance).

In meeting our requirements of a large gauge size, an important consideration is the temporal coherence of the source. Specifically, we require that the source have sufficient temporal coherence that, for a given point on the sensor, all points on the object are spatially coherent. Figure 2 shows that this requirement is satisfied when the path difference is less than the coherence length $l_{c}$ of the source,

$L_{1}-L_{2}<l_{c}$.

In the paraxial approximation, this requirement yields

$D \sin \theta<l_{c} \sim \lambda^{2} / \Delta \lambda$,

where $\Delta \lambda$ is the laser linewidth. This is a subtle requirement. It arises because we require that the light reflected from all points on the object be able to interfere (constructively and destructively) at any point on the detector. If this requirement is not met, then the effective gauge size is less than that of the illuminated spot. In fact, the estimate of the finest structure in the speckle pattern [Eq. (2)] is based on the assumption that the illumination over the disk of diameter $D$ is spatially coherent.

Finally, in implementing this measurement scheme, there are various physical phenomena that need to be addressed, such as atmospheric turbulence, vibration, and surface oxidation. We address these issues in Sec. 3.

\section{Data Processing Algorithm}

Conventional processing of one-dimensional speckle sequences has relied on computation of the correlation between the reference and sample speckle sequences. This approach is straightforward, and is actually strongly suggested by Yamaguchi's ${ }^{1}$ physical optics-based derivation of Eq. (1). For our application, however, this technique has some serious shortcomings. In particular, if the experiment is subject to vibration or turbulence, there will always be

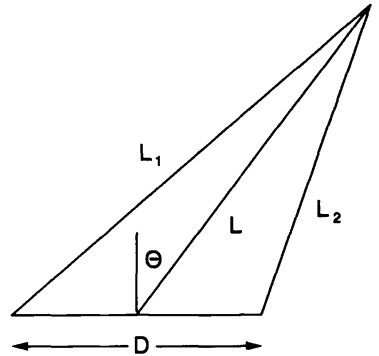

Fig. 2 Diagram showing temporal coherence required by measurement technique; requirement is satisfied when path difference is less than coherence length of the source.

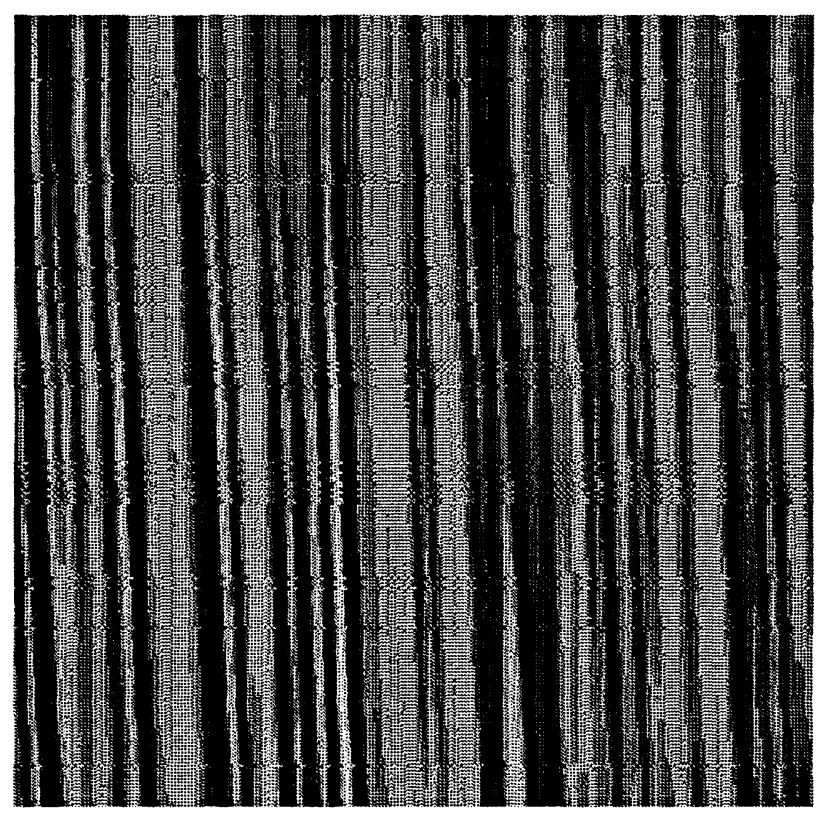

Fig. 3 Example of a stacked speckle history.

some question as to the validity of the reference exposure. Further, if the strains are substantial or the microstructure of the surface changes, for example as the result of oxidation, the speckle pattern will change and become decorrelated with the reference. Thus, a new reference must be chosen.

When we realize that the purpose of the conventional approach is simply to determine the lateral shift in a "noisy" pattern, however, a number of alternative processing schemes suggest themselves. Toward this end, we show in Fig. 3 what we call the "stacked speckle history" from a single camera. These data are for an object that is gradually strained from approximately $15 \mu \varepsilon$ at the top of the image to approximately $-15 \mu \varepsilon$ at the bottom, where each row represents a subset (240) of the total number of pixels. From Fig. 3 we can see that there is apparently some noise owing to timing irregularities in the camera, as well as a lateral shift in the speckle sequence and a gradual decorrelation in the speckle patterns as one proceeds from top to bottom.

The visual similarity between the data displayed in Fig. 3 and the appearance of unprocessed synthetic aperture radar (SAR) data ${ }^{12}$ is striking. The analogy is particularly strong between the "fast" (range) axis and the "slow" (Doppler) 


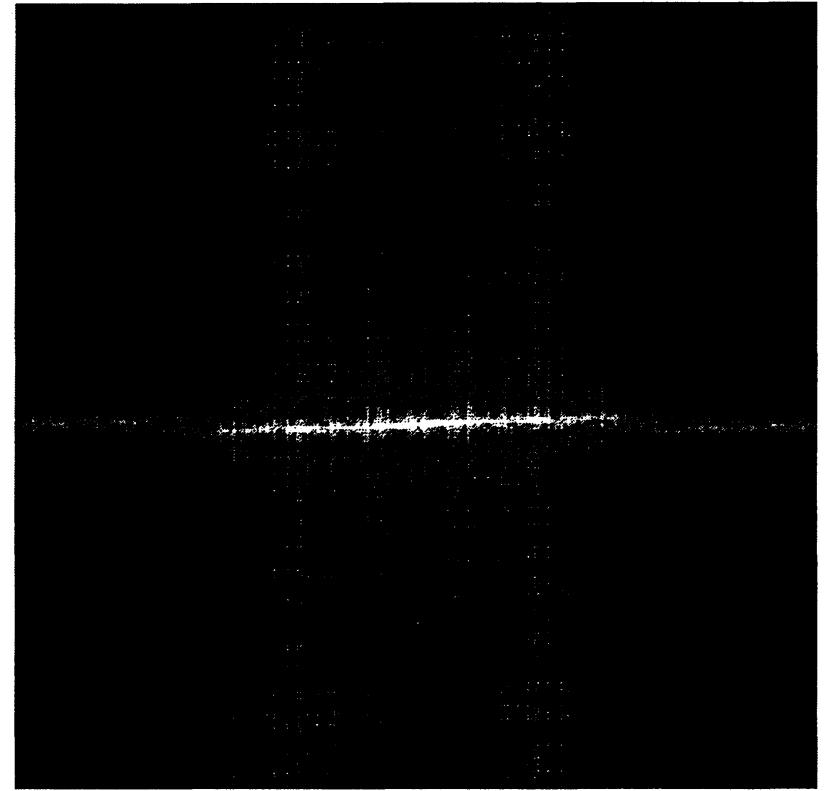

Fig. 4 Two-dimensional Fourier transform of stacked speckle history (logarithmic encoding). Spatial frequency is along the horizontal axis, and temporal frequency is along the vertical axis.

axis for the SAR data, and the "fast" (speckle) direction and the "slow" (temporal) direction. Naturally, the question arises as to how the desired information manifests itself in this display. Of course, the answer is simply that the time rate of speckle pattern shift is given by the tilt of the corrugated structure. The standard method of processing SAR data therefore suggests a simple method of processing: a two-dimensional Fourier transform implemented with a fast Fourier transform (FFT) algorithm. Figure 4 is an example of such an operation. In Fig. 4, spatial frequency is along the horizontal axis and temporal frequency is along the vertical; dc is in the center. The information that we desire is given simply by the slope of the bright line running through dc. From Eq. (1), we now have

$\delta \dot{A}_{x}=-2 L \dot{\varepsilon}_{x x} \tan \theta-2 \dot{a}_{z} \sin \theta$

where the time derivatives are indicated explicitly by the dot over the character. The term on the left-hand side of Eq. (5) is the time rate of the differential speckle pattern shift, which is simply the difference of the slopes, $m_{1}-m_{2}$, calculated from the two cameras. Under the assumption that the term containing the time rate of out-of-plane motion, $\dot{a}_{z}$, can be made negligibly small, Eq. (5) can be inverted to provide the estimate of the time rate of strain:

$\dot{\varepsilon}_{x x}=\frac{\delta \dot{A}_{x}}{-2 L \tan \theta}=\frac{m_{1}-m_{2}}{-2 L \tan \theta}$

Figure 5 summarizes our data-processing algorithm for estimating this time rate of strain.

To address the problem of vibration and/or turbulence we note that these are zero-mean processes. If we process our data over a period that is long compared to the period

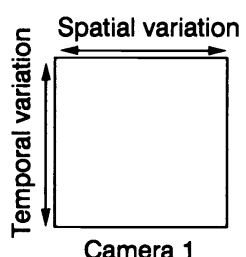

Speckle hlstorles

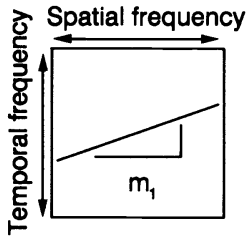

Transform domaln

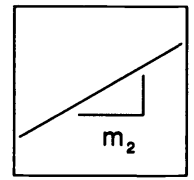

Fig. 5 Summary of data processing algorithm used to estimate time rate of strain.

of the noise process (which simply causes the speckle pattern to shift back and forth), the noise will be strongly suppressed.

Another benefit of this approach is the following: As suggested by Eq. (2), the finest structure in the speckle pattern, as measured at the detector, is given by the size of the illuminated spot, that is, the gauge size. Conversely, the largest scale sizes in the measured intensity are related to the microstructure of the surface. In other words, there is an uncertainty (Fourier transform) relationship between the scale sizes in the subject and observation planes. Our processing algorithm, which performs another Fourier transform, inverts this relationship so that the high spatial frequencies (far from the dc value; see Fig. 4) are related to the large-scale sizes on the subject, that is, the gauge size. Further, the low spatial frequencies are related to the fine structure of the subject's surface. From this, one can conclude that if the subject is straining equally at all scale sizes, then the bright band in the focused "image" will be a straight line. Any deviation from a straight line suggests that the subject is not straining equally at all scale sizes. Although this is not an imaging technique, it does provide global estimates (over the region being observed, set by the size of the illuminated spot) of the time rate of strain at a particular scale size.

Now the reason for the Nyquist criterion becomes apparent. The highest spatial frequency (cycles/length) in the transform domain, corresponds to $1 / \sigma$ (i.e., $D / \lambda L$ ). The spatial frequency resolution is $\Delta f_{s}=1 / N_{s} p$, where $p$ is the detector pixel pitch and $N_{s}$ is the number of points in the spatial FFT. In the spatial frequency dimension, the focused line image therefore will occupy $N=(2 / \sigma) / \Delta f_{s}=2 D N_{s} p / \lambda L$ pixel elements. This line image extends the full width of the image when $N=N_{s}$, that is, when $p=\lambda L / 2 D$. This is simply the Nyquist criterion, which we must meet to avoid aliasing the final focused line image. This brings up one final advantage of our processing approach. In conventional processing techniques based on computing correlations, the strain resolution is determined by the relative size of the speckles and the camera pixels. This puts severe limitations on the hardware (one needs small pixels) and the experimental configuration (one is restricted to small gauge sizes). With our technique, the resolution requirements are rather modest: we require only that the camera resolve the speckle structure. 


\section{Results}

Each of the two cameras used in our measurements was from I2S S.A. (model iDC161-BC-8). These are linear CCD arrays composed of 3456 square pixels, $10.7 \mu \mathrm{m}$ on a side (total array length is $36.98 \mathrm{~mm}$ ). Control of each camera was via a card plugged into a IBM AT personal computer. This configuration allowed software control of integration time and external triggering of the exposure. Data were acquired at 8-bit resolution.

For this experiment, the observation distance was $0.92 \mathrm{~m}$ and the off-axis angles were $\pm 32.5 \mathrm{deg}$. An illuminated spot measuring 1.46 by $0.387 \mathrm{~cm}$ was provided, using a LaserMax diode laser Model LAS 200-670-5 with an output of $4.5 \mathrm{~mW}$ and an optical bandwidth of $<0.1 \AA$. The resulting coherence length at $670 \mathrm{~nm}$ was at least $4 \mathrm{~cm}$. Since the path difference [Eq. (4)] was on the order of $9 \mathrm{~mm}$, the $1.59-\mathrm{cm}$ dimension did indeed constitute the gauge size. The minimum speckle size [see Eq. (2)] was approximately $39 \times 194 \mu \mathrm{m}$, or about $4 \times 18$ pixels. Illumination with a stripe instead of a circular spot was found to provide better contrast when using linear detectors.

The specimen being monitored was a piezoelectric ceramic made of lead zirconate-titanate (PZT), measuring $2 \times 1.5 \times 0.0762 \mathrm{~cm}$ (Vernitron material PZT-5A, with relevant piezoelectric constant $d_{31}=-171 \times 10^{-12} \mathrm{~m} \mathrm{~V}^{-1}$ ). Silver electrodes were attached to the large faces of the PZT specimen so when voltage was applied in the range of $\pm 70 \mathrm{~V}$, an electric field was established across its thinnest dimension. The strains produced along the long axis of the specimen are therefore given by the formula

$\varepsilon_{x x}=-d_{31} V / t$

where $V$ is the applied voltage and $t$ is the thickness of the specimen. Peak strains were $\pm 15.7 \mu \varepsilon$.

A Micro-Measurements precision strain gauge (model EQ-06-125AD-120-LE) was epoxy bonded to the back of the PZT specimen so that simultaneous conventional strain estimates could be made. This instrument was a $120-\Omega$ device with a 2.10 -gauge factor at $75^{\circ} \mathrm{F}$, and a gauge length of approximately $0.318 \mathrm{~cm}$. The total area covered by the gauge was approximately $0.318 \times 0.318 \mathrm{~cm}$. Strain measurements were obtained with a Measurements Group P3500 strain indicator.

During the course of the experiment, a sinusoidal voltage with a frequency of $3 \mathrm{mHz}$ was applied to the PZT specimen. The cameras were sampled every $3 \mathrm{~s}$, and the strain gauge readings and the applied voltages were recorded using a Keithley DAS-8PGA digital-to-analog (D/A) card mounted on the personal computer bus.

To illustrate our approach, a subset of the cameras' output is shown in Fig. 6. These figures are sequences of 175 exposures each of 512 pixels. The strain gauge readings are shown in Fig. 7. A linear regression fit to the strain as a function of voltage produced the results in Fig. 8, with an estimated strain coefficient of $-0.216 \mu \varepsilon V^{-1}$. Figure 9 shows a subset of 10 exposures used in estimating strain rate based on our image-processing scheme. These data points correspond to the peak strain rate produced $(0.276$ $\mu \varepsilon \mathrm{s}^{-1}$ ). (This estimate is based on a complete 20 -min set of strain gauge data; if only the 30 -s period is used, the strain rate estimate is $0.230 \mu \varepsilon \mathrm{s}^{-1}$ ). Figure 10 shows the results of computing the Fourier transforms of the $512 \times 10$ data sets and subsequently peak detecting the data. To compute these transforms, a $512 \times 256$ FFT was used with zero filling in the temporal direction. Figure 10 also shows linear regression fits to the focused line images. From the estimates of the linear slopes, the resulting estimate of the strain rate [see Eq. (6)] is $0.304 \mu \varepsilon \mathrm{s}^{-1}$.

The data in Fig. 10 suggest that there may be some curvature in the focused line. Figure 11 therefore shows results for a second-order fit. This procedure produced a higher strain rate estimate (for the fine-scale structure) of $0.511 \mu \varepsilon \mathrm{s}^{-1}$. Of course, these second-order fits produce local slope estimates (and thus strain rates) that are dependent on spatial frequency, $m_{1}\left(f_{s}\right)$ and $m_{2}\left(f_{s}\right)$.

\section{Discussion and Conclusions}

We have developed a highly sensitive method for measuring rates of thermal expansion, mechanical strain, and creep. This technique is based on Yamaguchi's speckle strain gauge concept, but uses a novel data-processing approach that is reminiscent of synthetic aperture radar processing.

Our approach has numerous advantages. Although not an imaging technique (the processed "image" is actually the power spectral density of the stacked speckle history), it does provide simultaneous global strain rate estimates at all scale sizes. To the extent that different creep mechanisms take effect at different length scales, our technique may be able to distinguish the mechanisms in the midst of a test, before failure. For example, it would be beneficial to differentiate between intragranular and transgranular cracking to obtain better input into predictive models. ${ }^{13}$ Further advantages include large gauge sizes, compact design, insensitivity to zero-mean noise processes such as turbulence and vibration, and modest requirements for pixel resolution. We base our claim of insensitivity to vibration and turbulence on the fact that the slopes, and thus the strain estimates, are calculated over an ensemble of speckle patterns rather than on a pair, as in conventional correlation techniques. The variance of these estimates thus can be expected to decrease inversely with the number of samples.

We are currently exploring the use of parametric (e.g., autoregressive $^{14}$ and Bayesian ${ }^{15}$ ) spectral estimation techniques in the temporal dimension. The advantage of parametric spectral estimation techniques is that they make use of $a$ priori knowledge of the process. Specifically, we know in this case that the focused line image will be highly localized. After the nonparametric transformation in the spatial direction (using the FFT), we know that transformation in the temporal direction should produce a narrow "line", spectrum at each spatial frequency. The nonparametric spectral estimator used herein, while producing accurate strain rate estimates in this example, does not take advantage of this a priori knowledge. Furthermore, parametric techniques are particularly appropriate for processes displaying a lack of stationarity (see Fig. 3). These techniques also promise increased resolution in the temporal dimension. This is especially important because at small strain rates the estimate of the slope of the focused line image is particularly sensitive to the resolution of the spectral estimator used in the temporal direction.

In the example presented herein, our results were based on $30 \mathrm{~s}$ of data and the estimated strain rates were on the 


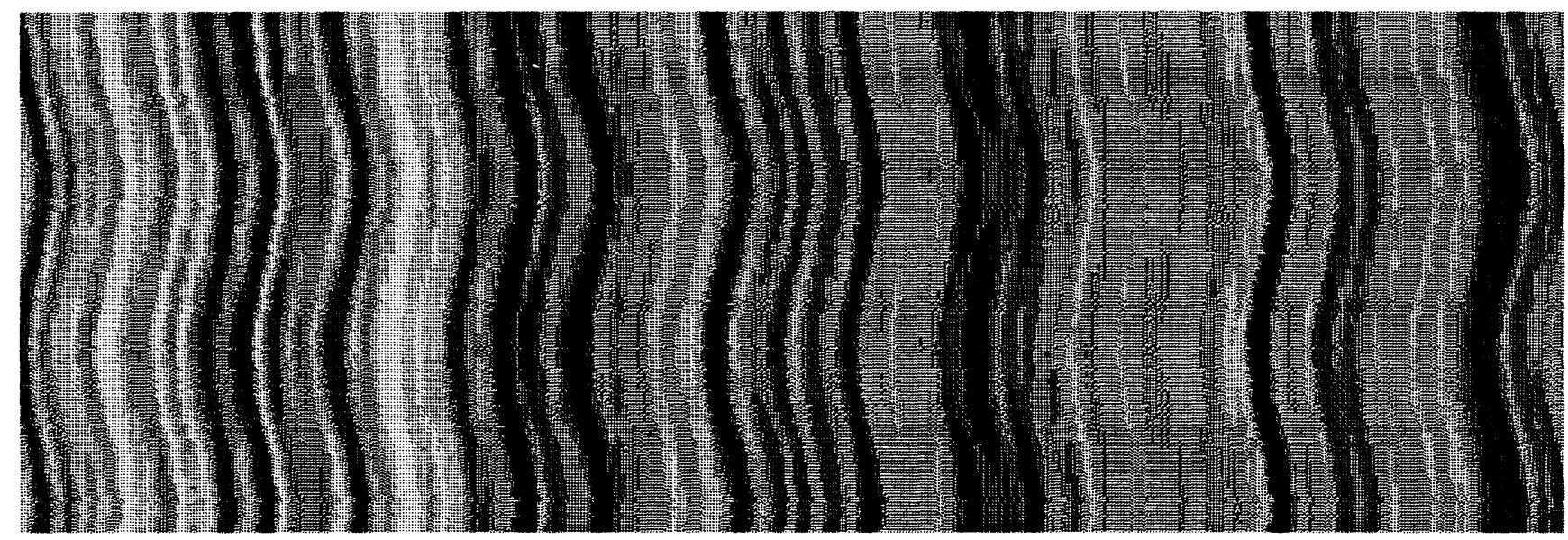

(a)

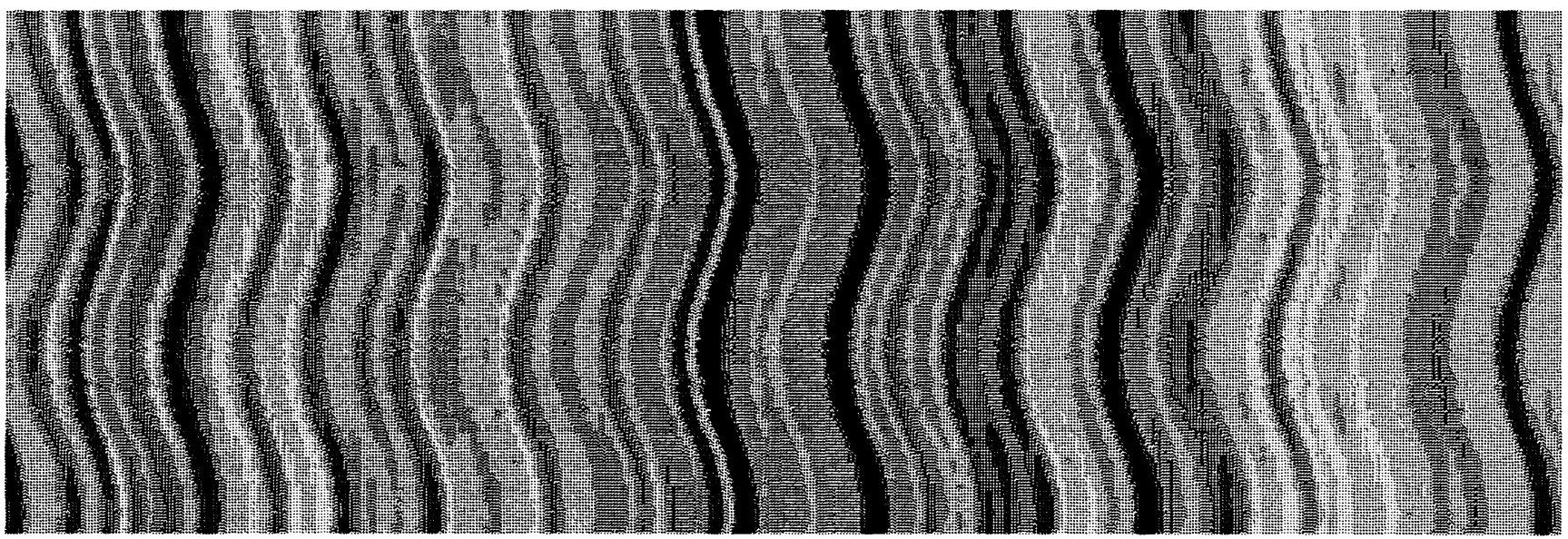

(b)

Fig. 6 Stacked speckle histories for sinusoidal voltage applied to PZT specimen: (a) camera 1 and (b) camera 2.

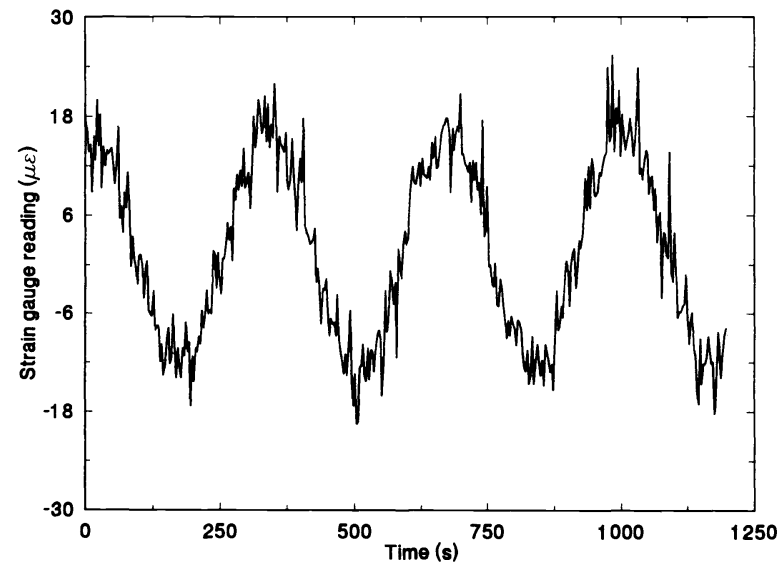

Fig. 7 Strain gauge estimate.

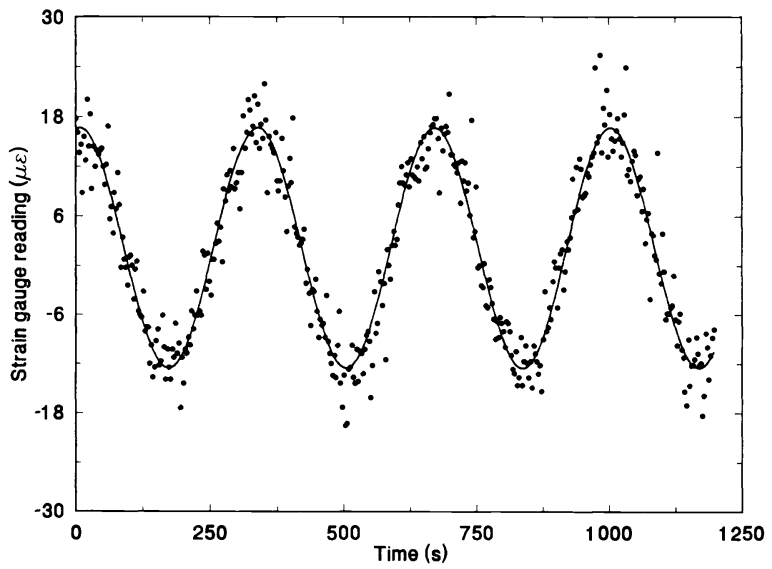

Fig. 8 Result of linear regression fit to strain gauge data. 


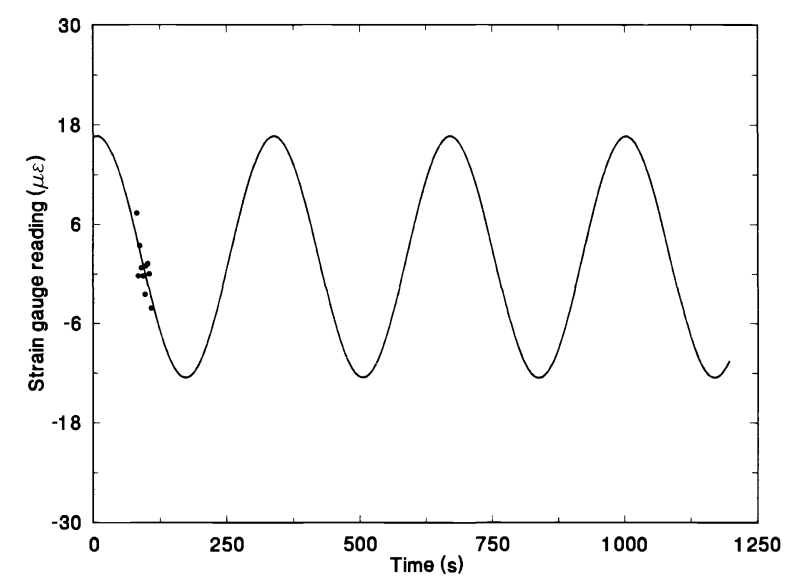

Fig. 9 Subset of data showing 10 exposures used in estimating strain rate, based on image-processing algorithm.

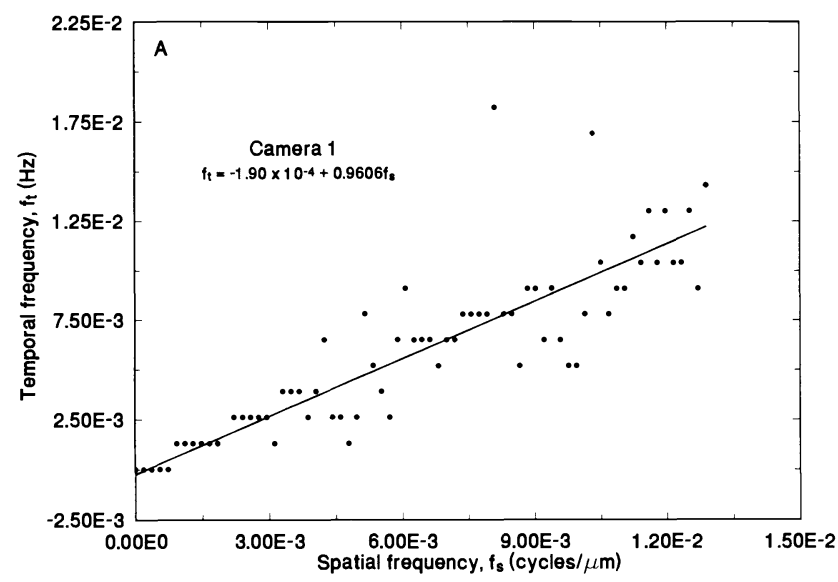

(a)

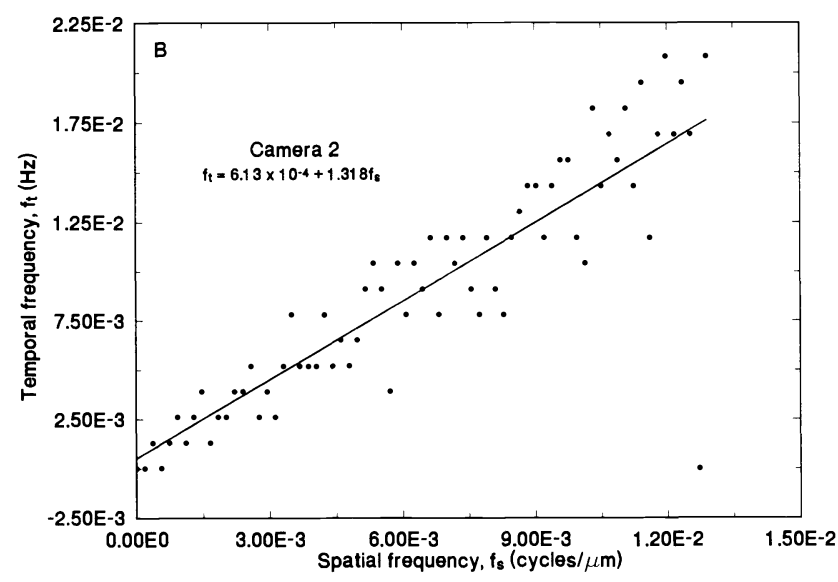

(b)

Fig. 10 Peak-detected focused line "images" obtained using imageprocessing algorithm: (a) camera 1 and (b) camera 2 .

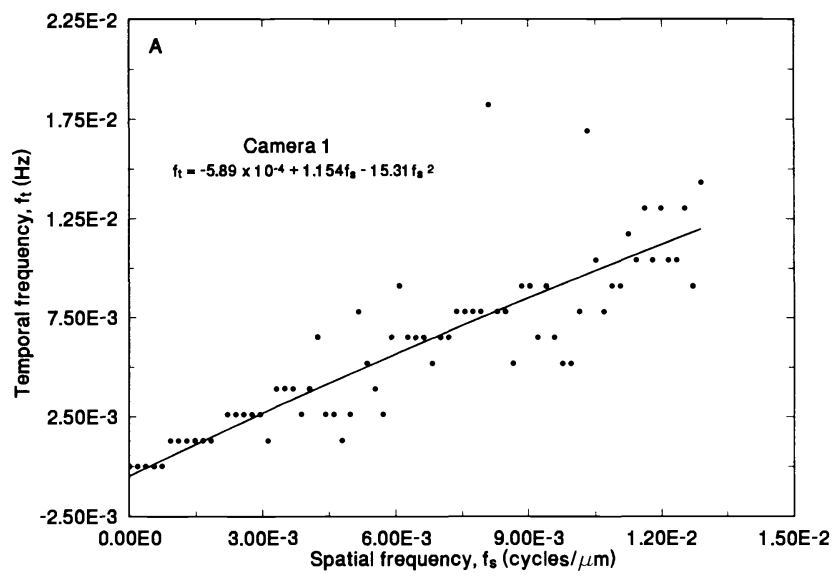

(a)

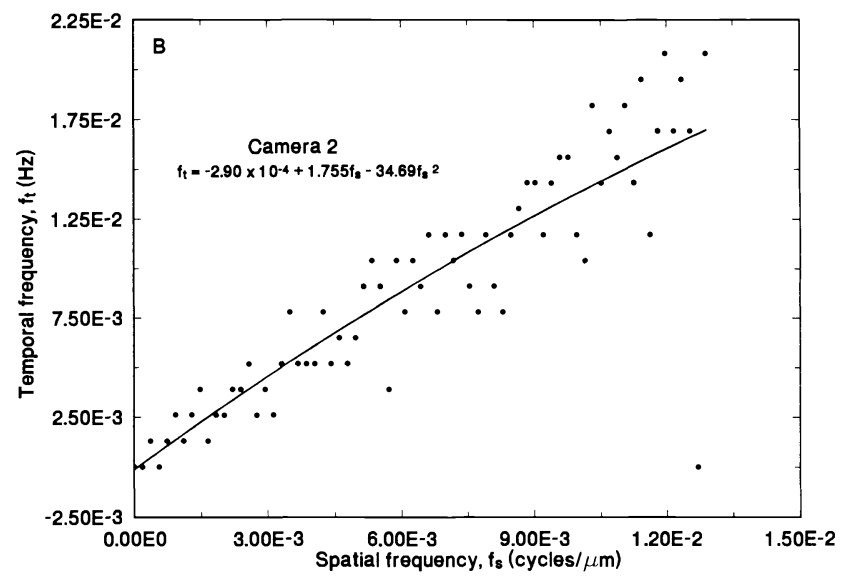

(b)

Fig. 11 Second-order fits to focused line images: (a) camera 1 and (b) camera 2.

order of $0.3 \mu \varepsilon \mathrm{s}^{-1}$. The total strains over the observation period were therefore about $9 \mu \varepsilon$. Based on Eq. (1), the resolution on strain measurement is dictated by the camera pixel spacing. For our experiment, the minimum observable strain using a correlation-matching technique, therefore, would have been about $9 \mu \varepsilon(p / 2 L \tan \theta=9.11 \mu \varepsilon)$. Our goal is to be able to estimate strain rates based on total observed strains on the order of $1 \mu \varepsilon$. This would allow measurement times of no longer than $1 \mathrm{~h}$ (for polycrystalline alumina). Preliminary tests using parametric spectral estimation techniques suggest that this should be possible. The fact that these strains were produced over a period of $0.5 \mathrm{~min}$ is irrelevant. They could just as easily have been generated over a period of hours. For longer-term experiments of this kind, the sample interval would be altered accordingly.

\section{Acknowledgment}

We gratefully acknowledge the internal R\&D support for this work from the Applied Physics Laboratory of the Johns Hopkins University. 


\section{References}

1. I. Yamaguchi, "Speckle displacement and decorrelation in the diffraction and image fields for small object deformation," Optica Acta 28(10), 1359-1376 (1981).

2. M. Françon, Laser Speckle and Applications in Optics, H. H. Arsenault, trans. Academic Press, New York (1979).

3. R. K. Erf, Ed., Speckle Metrology, Academic Press, New York (1978).

4. D. Joyeux and S. Lowenthal, "Real time measurement of angstrom order transverse displacement of vibrations, by use of laser speckle,' Opt. Commun. 4(2), 108-112(1971).

5. I. Yamaguchi, "Simplified laser-speckle strain gauge," Opt. Eng. 21(3), 436-440 (1982).

6. I. Yamaguchi, "Advances in the laser speckle strain gauge," Opt. Eng. 27(3), 214-218 (1988).

7. J. P. Barranger, "Two-dimensional surface strain measurement based on a variation of Yamaguchi's laser-speckle strain gauge," NASA Technical Memo. 103162, National Aeronautics and Space Administration, Lewis Research Center, Cleveland, Ohio (1990).

8. T. Takemori, K. Fujita, and I. Yamaguchi, "Resolution improvement in speckle displacement and strain sensor by correlation interpolation," in Laser Interferometry IV: Computer-Aided Interferometry, Proc. SPIE 1553, 137-148 (1991).

9. A. Oulamara, G. Tribillon, J. Duvernoy, "Subpixel speckle displacement measurement using a digital processing technique," J. Modern Opt. 35(7), 1201-1211 (1988).

10. J. W. Goodman, "Statistical properties of laser speckle patterns, in Laser Speckle and Related Phenomena, J. C. Dainty, Ed., pp. 9-75, Springer-Verlag, New York (1975).

11. A. W. Greynolds, "A consistent theory of scatter from optical surfaces," in Stray Light and Contamination in Optical Systems, Proc. SPIE 967, 10-18 (1988).

12. J. P. Fitch, Synthetic Aperture Radar, Springer-Verlag, New York (1988).

13. D. W. Richerson, Modern Ceramic Engineering: Properties, Processing and Use in Design, Marcel Dekker, Inc., New York (1982).

14. S. L. Marple, Jr., Digital Spectral Analysis with Applications, PrenticeHall, Inc., Englewood Cliffs, N.J. (1987).

15. G. L. Bretthorst, Bayesian Spectrum Analysis and Parameter Estimation, Springer-Verlag, New York (1988).

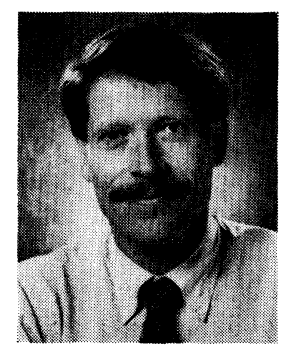

Donald D. Duncan received a BS in electrical engineering from the University of Kentucky in 1970, and an MS in 1973 and a PhD in 1977 in electrical engineering from The Ohio State University. He is a member of OSA and Sigma Xi. He worked on propagation through turbulent and aerosol laden media, laser metrology, and signal processing for Pacific-Sierra Research Corporation in Dayton, Ohio, and Rosslyn, Virginia, and is currently pursuing those interests at the Johns Hopkins University Applied Physics Laboratory in Laurel, Maryland.

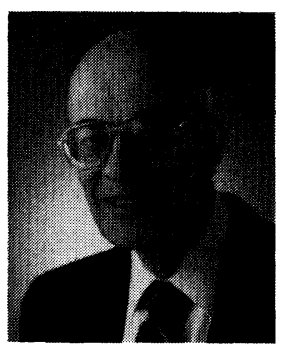

F. Fausten Mark studied physics at MIT and is a member of the Advanced Systems Group of the Aeronautics Department at the Johns Hopkins University Applied Physics Laboratory. His work is in the fields of fluid dynamics, biomedical research, optics, and advanced materials.

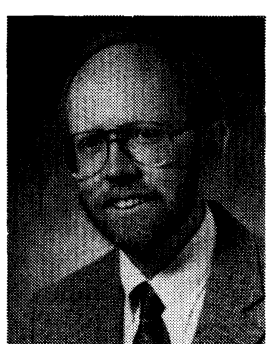

Lawrence W. Hunter received a PhD degree in theoretical chemistry from the University of Wisconsin in 1971 and subsequently joined the staff of the Johns Hopkins University Applied Physics Laboratory. Hunter's publications include articles on molecular collision theory (with applications to gas property calculations), combustion and fire problems, and methods for calculating aerodynamic ablation rates of materials. He is a member of AIAA, the American Ceramic Society, and the Combustion Institute. 